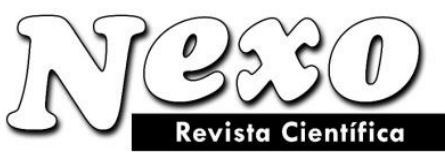

Vol. 32, No. 01, pp. 27-40/Junio 2019

\title{
AGUAS DE CONSUMO HUMANO Y CALIDAD DE VIDA: CASO COMUNIDAD "EL CACAO" MOZONTE, NUEVA SEGOVIA
}

\section{WATERS OF HUMAN CONSUMPTION AND QUALITY OF LIFE: CASE COMMUNITY "EL CACAO", MOZONTE, NUEVA SEGOVIA}

\author{
L.A. Páramo-Aguilera ${ }^{1 *}$ y P. Rivas-Pérez² . \\ ${ }^{1}$ Programa de Investigación Estudios Nacionales y Servicios del Ambiente (PIENSA), Universidad \\ Nacional de Ingeniería (UNI), Avenida Universitaria, Fte a la UCA, Managua, Nicaragua. \\ ${ }^{2}$ Facultad de Ciencias Económicas y Empresariales, UCA. Frente a Radio ya, Managua, Nicaragua. \\ *Iparamo2014@gmail.com, rivaspatricia@doc.uca.edu.ni
}

(recibido/received: 22-Febrero-2019; aceptado/accepted: 14-Mayo-2019)

\section{RESUMEN}

La comunidad El Cacao, municipio de Mozonte, departamento de Nueva Segovia está ubicada a $234 \mathrm{~km}$ de la capital Managua. En invierno se alimentan del agua de arroyos y quebradas, mientras que en verano se alimentan de un ojo de agua donde se han construido algunas instalaciones mínimas para el almacenamiento y distribución. Estudios fisicoquímicos, microbiológicos, de plaguicidas y metales pesados; determinaron la presencia de diversos tipos de contaminantes de naturaleza química y principalmente microbianos en estas aguas. Se determinó que 123 personas hacen uso de la fuente en estudio (54\% adultos, $43 \%$ niños y $3 \%$ ancianos). Las encuestas aplicadas a 24 jefes de familia de la comunidad El Cacao, mostraron una relación entre la educación sanitaria y la calidad del agua, ya que los pobladores carecen del conocimiento de normas higiénico sanitarias orientadas al mejoramiento de las condiciones de vida. Evidenciando así la relación existente entre nivel educativo y niveles de ingreso por familia con las condiciones de salud observadas y su relación con el manejo del agua desde el acuífero hasta el hogar. Concluyéndose que la esperanza de vida de las personas en esta comunidad es baja y está por debajo del valor nacional (75.1 años).

Palabras claves: Agua potable; Saneamiento; Calidad de vida; Educación sanitaria y calidad de agua.

\section{ABSTRACT}

El Cacao Community, municipality of Mozonte, Nueva Segovia department is located to $234 \mathrm{~km}$ from the capital Managua. In winter they feed of the water of creeks and gullies, while in summer they feed of a water eye where there are some minimum installations for storage and distribution. Physicochemical studies, microbiological, pesticide and wave metals; determined the presence of diverse types of pollutants from chemically nature principally microbiological in these waters. It has shown that 123 persons use the source in study (54\% adults, $43 \%$ child and $3 \%$ elders). The applied surveys to 24 family heads form El Cacao Community, show a relation between sanitary education and quality of the water, because the people lack of the knowledge of the hygienic sanitary procedure orientated to improvement of the living conditions. Demonstrating this way, the existing relation between educational level and the family's income levels with 


\section{L.P. Páramo-Aguilera y P. Rivas-Pérez}

the conditions of health observed and their relation with the water management form the source until the home. Concluding that the life expectancy of the persons in this community is low and this one below the national value (75.1 years).

Keywords: Drinking water; Sanitation; Quality of life; Health education and water quality.

\section{INTRODUCCIÓN}

Uno de los factores contaminantes más importantes del agua es de origen antropogénico, ya sea por ciertas actividades que se llevan a cabo cercanas a las fuentes de agua, así como de las condiciones higiénicas sanitarias en las que viven los pobladores de la comunidad. Más de un billón de personas alrededor del mundo consumen agua contaminada, y cada año 3.4 millones de estas, principalmente niños, mueren a causa de enfermedades de transmisión hídrica; de estas muertes, 2.2 millones son causadas por enfermedades diarreicas. El 90\% ocurren en los niños principalmente en países en vías de desarrollo (Valiente y Mora, 2002). Históricamente, el papel del agua para consumo humano ( $\mathrm{ACH})$ ha sido fundamental, tanto en la prevención como en la transmisión de agentes causantes de diarreas y otras enfermedades como hepatitis A, polio y parasitosis por protozoarios y helmintos; dentro de estas tenemos las amebiasis, giardiasis, cryptosporidiasis y helmintiasis, como la provocada por el Ascaris lumbricoides. La diferencia entre prevenir o transmitir este tipo de enfermedades de origen hídrico, depende de varios factores, los principales son la calidad y la continuidad del servicio. El ACH participa en la transmisión de enfermedades por medio de cuatro vías: la primera es la ingesta de microorganismos patógenos o de sustancias químicas tóxicas; la segunda se da por contacto directo con el agua contaminada (higiene personal, natación); la tercera es la relacionada con la ausencia o escasez del agua para la higiene personal, lo cual favorece la transmisión orofecal (ano-mano-boca) y colabora en la exacerbación de otras enfermedades de la piel, ojos y oídos, y la última es la transmisión de enfermedades por vectores acuáticos (Dengue, Malaria, etc.) (McJunkin, 1986).

De acuerdo a Pérez (2007), la educación sanitaria y ambiental se está convirtiendo en uno de los propósitos más relevantes para el mejoramiento de las condiciones de vida de las personas, ya que se debe estar consciente del cuidado de la salud; por ello, no se puede entender el desarrollo social, el desarrollo de las familias y comunidades sin el enfoque del cuido y mejoramiento de los estilos de vida y de los hábitos de higiene. Según la Organización Mundial de la Salud (2006 y 2007), el agua en las zonas rurales se contamina debido a una manipulación poco higiénica durante su transporte o en casa, es por ello que es necesario tomar ciertas medidas higiénico-sanitarias en las actividades de recepción, almacenamiento y usos del agua.

La comunidad El Cacao está ubicada en el municipio de Mozonte del departamento de Nueva Segovia. Este municipio está ubicado a $234 \mathrm{~km}$ de la ciudad capital Managua. Su posición geográfica se encuentra entre las coordenadas $13^{\circ} 39^{\prime \prime}$ de latitud norte y $86^{\circ} 26^{\prime \prime}$ de longitud oeste, a 693 metros sobre el nivel del mar (m.s.n.m.), con una extensión territorial de $242 \mathrm{~km}^{2}$. Limita al norte con Honduras, al sur con el municipio de Totogalpa, al este con el municipio de San Fernando, Ciudad Antigua y Telpaneca y al oeste con el municipio de Ocotal y Dipilto. El Cacao se ubica en la zona sur del municipio de Mozonte, a 5,9 km de la ciudad de Ocotal, cabecera departamental de Nueva Segovia (INIDE, 2010). Se abastece de agua proveniente de pozos y fundamentalmente de un ojo de agua, a través de un sistema instalado que inicia con una pila de captación ubicada en el ojo de agua, su conducción a un tanque de almacenamiento ubicado aguas abajo del sitio de captación y finalmente una llave de pase (de chorro), la cual es el punto de recolección del agua por parte de la población, pero este sistema no cuenta con ningún tipo de tratamiento para potabilizar el agua.

Cabe mencionar que 24 familias (123 personas), de los cuales 66 son personas adultas que equivalen al $54 \%$; 53 niños que representan un equivalente a $43 \%$ y tan solo 4 ancianos que representan un 3\% de adultos mayores de la comunidad se benefician del sistema antes descrito, pero en los últimos tiempos se ha observado la aparición de enfermedades en la piel de la población, enfermedades entéricas y otras que han

Nexo Revista Científica / Vol. 32, No. 01, pp. 27-40/Junio 2019 
motivado la realización de este trabajo; como un esfuerzo conjunto de la Alcaldía de Mozonte, la comunidad El Cacao y el Programa de Investigación Estudios Nacionales y Servicios Ambientales de la Universidad Nacional de Ingeniería (PIENSA-UNI). Gobierno local, academia y población, unen esfuerzos en pro de buscar las causas que están motivando la aparición de estas enfermedades en la población y su posible relación con las condiciones del agua que consumen, su transportación y almacenamiento en los hogares con la calidad de vida de los lugareños.

\section{METODOLOGÍA}

\subsection{Ubicación de las tomas de muestras}

Las muestras para análisis se tomaron en tres puntos de muestreo descritos en la Tabla 1 y Figura 1 que se muestra a continuación:

Tabla 1. Descripción de los puntos de muestreos. Punto 1. Pila de captación, Punto 2. Tanque de almacenamiento, Punto 3. Llave de chorro. Estos puntos se pueden apreciar en la figura 1.

\begin{tabular}{cl}
\hline Puntos de muestreo & \multicolumn{1}{c}{ Ubicación } \\
\hline Punto 1 & $\begin{array}{l}\text { Pila de captación ubicada en el ojo de } \\
\text { agua }\end{array}$ \\
Punto 2 & $\begin{array}{l}\text { Tanque de almacenamiento ubicado } \\
\text { aguas abajo del sitio de captación }\end{array}$ \\
Punto 3 & $\begin{array}{l}\text { Llave de chorro ubicada en el punto de } \\
\text { recolección del agua por parte de la } \\
\text { población }\end{array}$ \\
\hline
\end{tabular}
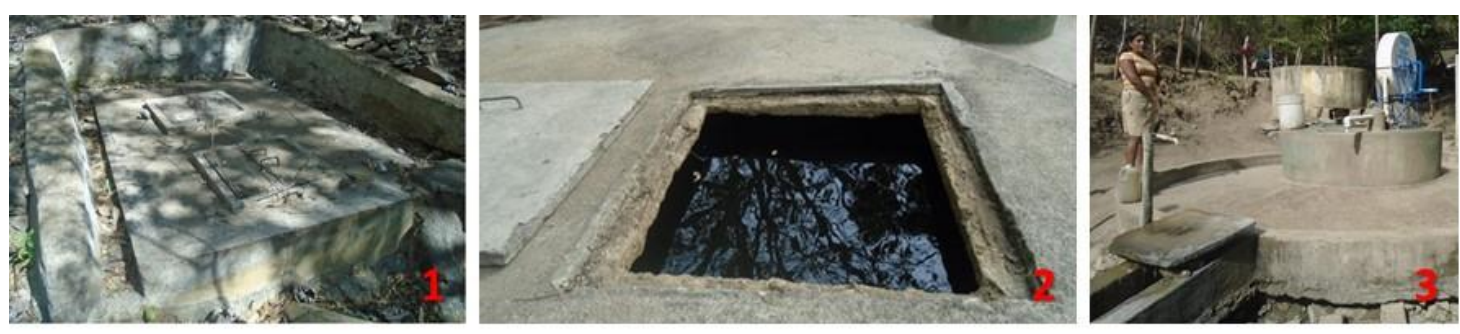

Figura 1. Se muestran los diferentes puntos monitoreados en la comunidad El Cacao.1. Pila de captación, 2. Tanque de almacenamiento, 3. Llave de chorro

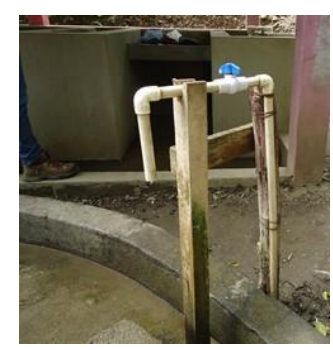

Figura 2. Se observa la llave de chorro de donde la población recoge el agua en cubetas y pichingas plásticas. Al fondo de la llave de chorro se observan áreas de lavado de ropa. Estas pilas del área de lavado, son llenadas a su vez por el reboso del agua desde el tanque principal de almacenamiento. 
Los puntos de muestreo previamente descritos (Tabla 1 y Figura 1), constituyen los puntos en donde inicialmente se recolecta el agua en una pila de captación construida en el ojo de agua con el propósito de recolectar el flujo de agua que sale que es sumamente pequeño (Punto 1). Posteriormente y una vez que se alcanza un nivel determinado en la pila, el flujo de agua fluye unos 250 metros aguas abajo por un sistema de tuberías de PVC de 2 pulgadas hasta llegar a un tanque de recolección (punto 2), en donde se espera que se alcance un volumen determinado que permita que se pueda suministrar agua a la población al menos día por medio. Del tanque de recolección el agua es enviada a una llave de chorro de media pulgada (Punto 3 y Figura 2), en donde la población acude a llenar sus recipientes que posteriormente traslada individualmente a sus hogares sin haber sido sometida a ningún sistema de tratamiento. Una vez que es llenado el tanque, por reboso del mismo, el agua es trasladada a otras pilas en donde la población realiza labores de lavado de ropa.

\subsection{Encuesta para determinar el impacto social de la calidad del agua}

Para determinar el impacto social de la calidad del agua en la comunidad El Cacao se elaboró y se aplicó una encuesta casa por casa (Figura 3, encuesta no mostrada en este artículo y que puede ser vista en Garmendia-Almendarez y Villalta-Domínguez, 2017), para ello se entrevistaron a 24 jefes de familia que toman agua del acuífero (100\% de las familias que hacen uso de esta fuente de agua), con el objetivo de conocer los datos específicos de las viviendas, los datos demográficos y el comportamiento del consumo del agua, haciendo énfasis en este último, para conocer la manera que almacenan el agua luego de ser recolectada, si hacen uso de algún proceso de desinfección del agua antes de consumirla y la cantidad promedio del agua utilizada en las labores cotidianas, entre otra información que permitió analizar las condiciones de vida de población, su condición socioeconómica asociado a su nivel de escolaridad y como toda esta información repercute en el uso y manejo del agua que los habitantes consumen. Una vez que se aplicó la encuesta los datos fueron procesados en el programa estadístico SPSS (Statistical Package for the Social Sciences) versión 23.0 para determinar la posible relación entre las condiciones higiénico sanitarias de los pobladores y la calidad del agua, entre muchas otras interrelaciones que fueron analizadas.
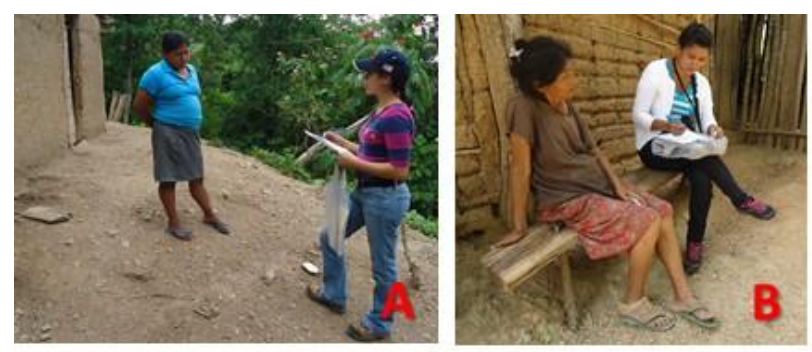

Figura 3. Se muestra el proceso de entrevistas aplicadas a los pobladores que toman agua del acuífero de la comunidad El Cacao. A y B, muestran el proceso de levantado de la información en dos viviendas diferentes.

\section{RESULTADOS Y DISCUSIÓN}

Según la GWP (Global Water Partnership -GWP) la gestión integrada del agua es un proceso que promueve la gestión y el aprovechamiento coordinado del agua, la tierra y los recursos relacionados, con el objetivo de maximizar el bienestar social y económico de manera equitativa sin comprometer el desarrollo sustentable de los ecosistemas vitales. Desde la perspectiva de la GWP, la gestión integrada del agua debe ser un proceso que comprometa tanto a las autoridades internacionales, gubernamentales, locales, al sector privado como a la sociedad entera, con el objetivo de evitar confrontaciones y prevenir desequilibrios en los ecosistemas. El enfoque de la GWP destaca no sólo una visión participativa sino también un compromiso en los modelos educativos de la gestión y utilización del agua (Nieto, 2011). 


\section{L.P. Páramo-Aguilera y P. Rivas-Pérez}

Uno de los factores contaminantes más importantes del agua es de origen antropogénico, ya sea por ciertas actividades que se llevan a cabo cercanas a las fuentes de agua, así como de las condiciones higiénico sanitarias en las que viven los pobladores de la comunidad. Dada la importancia que revisten el suministro de agua doméstica y los servicios de saneamiento para el bienestar social, los aspectos sociales de esta materia deben tenerse en cuenta a la hora de tomar cualquier decisión fundamental que pueda afectar a la prestación de servicios de agua $(\mathrm{OECD}, 2003)$. El desarrollo del trabajo condujo a los siguientes resultados:

\subsection{Selección de los puntos de toma de muestras}

La pila de captación es una pila con tapa que aísla al ojo de agua del ambiente, evitando de esta forma la entrada de basura, de cualquier suciedad y de los animales que merodean el lugar. Esta se encuentra ubicada al pie de uno de los cerros entre los cuales se ubica la comunidad El Cacao, en el municipio de Mozonte. El tanque de almacenamiento se encuentra ubicado a unos 250 metros aguas abajo en el sentido de un caño que está en el lugar y ahí se encuentran las instalaciones mínimas para la recepción del agua (Tanque de almacenamiento), pilas para lavado que se observan al fondo de la Figura 2 y un área de toma de agua en el que la población llena sus recipientes y los traslada personalmente hacia sus viviendas sin que el agua haya recibido algún sistema de tratamiento previo a su consumo. Un esquema que da una idea general del sistema tal cual se encuentra en el lugar, se esboza en la Figura 4.

El punto 1 mostrado en la Figura 1 y que corresponde a la pila de captación ubicada en el ojo de agua, reviste vital importancia porque en este punto se puede observar que el agua sale por el ojo de agua con presencia de rastros de una biopelicula (Figura 5) que en menos de 8 horas forma una capa gruesa en la superficie del agua y que viaja por el sistema de tuberías observándose su presencia tanto en tuberías, como en el tanque de recolección. Lo anterior hace suponer que es muy probable que la biopelicula viaje en forma planctónica (Estado libre o individual) desde la pila de captación hasta los hogares y que este hecho, este en estrecha relación con la aparición de distintas enfermedades que se observan en la población que habita en la comunidad. De la biopelicula se pudo aislar e identificar por vía molecular, diferentes microorganismos pertenecientes a hongos filamentosos del género Aspergillus, bacterias pertenecientes a diferentes géneros y especies como E. coli, Alcaligenes sp, Paenalcaligenes sp, Alcaligenes faecalis, Paenalcaligenes suwonensis, Proteus mirabilis, Serratia nematodiphilia y Stenotrophomona maltophilia (Páramo-Aguilera, et al., 2018). Es conocido que algunos de estos microorganismos están asociados con algunas enfermedades que se pudo observar en la población de la comunidad El Cacao.

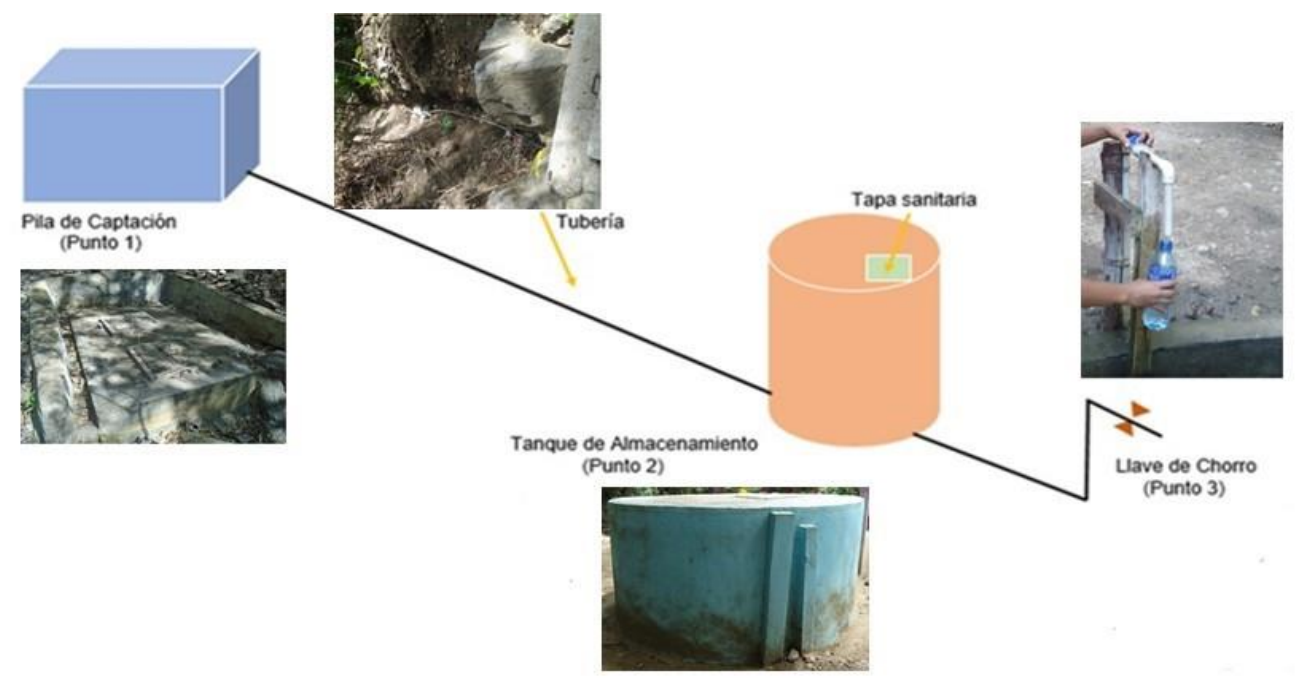

Figura 4. Esquema representativo de los componentes del sistema de recolección y distribución de agua de la comunidad El Cacao, haciendo uso de un ojo de agua ubicado en el punto 1. 


\section{L.P. Páramo-Aguilera y P. Rivas-Pérez}
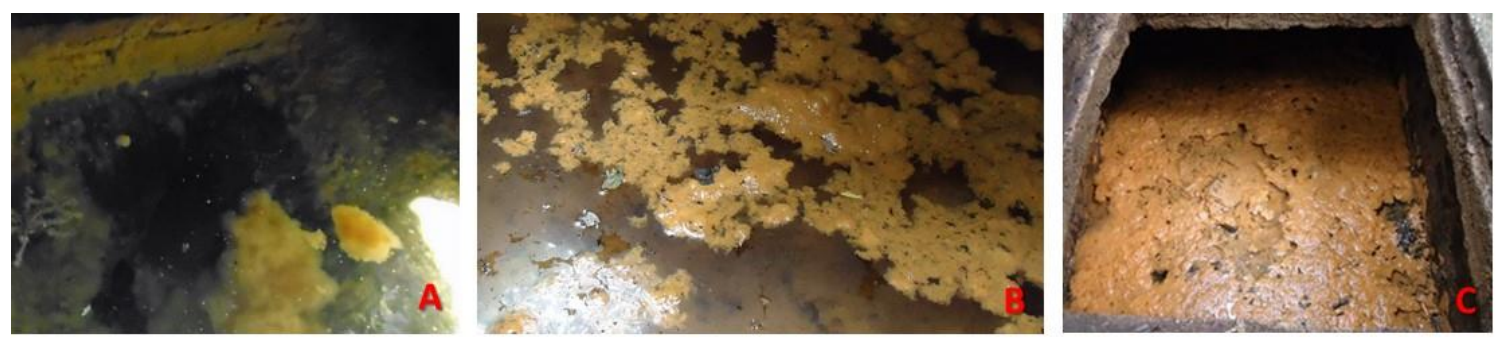

Figura 5. Se observan las diferentes etapas de formación de la biopelicula en la pila de captación. A. Comienza el llenado de la pila, inmediatamente después de lavada y retirada la biopelicula formada. B. Transcurridas unas 4 horas después de lavada la pila. C. Transcurridas unas 8 horas después de iniciado el proceso de llenado de la pila, la biopelicula ha cubierto completamente la pila y desde el paso B se da por iniciado el traslado del agua de la pila de captación al tanque de recolección.

3.2. Resultados obtenidos como producto de la aplicación de encuestas a la población que hace uso del agua de la fuente en estudio.

Actualmente, una de cada cinco personas (20\%) de la población mundial no tiene acceso al agua potable en el mundo, y se prevé que para el 2025 dos tercios de la población mundial, o sea 66.6\%, enfrentará problemas de insuficiencia de agua, y un tercio de la población global (33.3\%) vivirá en escasez absoluta. Para el 2030 se proyecta que uno de cada cinco países en el mundo enfrentará penurias de agua. Además, se espera que para el 2025 la falta del agua desencadene problemas de insuficiencia alimentaría de aproximadamente 350 millones de toneladas de alimento a escala mundial (Nieto, 2011).

Resultados demográficos: El conocimiento de los resultados demográficos para el área en estudio, resulta de gran interés, dado que esto permitirá que se tomen las mejores decisiones que contribuyan a mejorar las condiciones de vida de los habitantes y que a la vez considere su nivel socioeconómico para que no se convierta esta mejora de vida (al tener mejor acceso al agua) en un nuevo factor que tensione sus ya maltrechas economías familiares. Por ejemplo, un estudio sobre el mejoramiento del nivel de acceso al agua en México (OECD, 2003) mostró lo siguiente: Oaxaca, Chiapas y Guerrero tienen los niveles de servicios de agua más bajos y son los Estados más pobres en términos de desarrollo en general, en especial, desde el punto de vista de la "marginación". En los Estados de la Península de Yucatán también se dan niveles de conexión a la red pública de drenaje bajos, que probablemente puedan explicarse por las condiciones geológicas del lugar (esto es, ausencia de cursos de agua superficiales). Cabe destacar que el porcentaje de viviendas sin saneamiento de ningún tipo es mayor en Oaxaca (54\%), Guerrero (46\%) y Chiapas (37\%) que en los tres Estados de Yucatán: Campeche (36\%9, Quintana Roo (16\%) y Yucatán (41\%). Estos resultados nos muestran de manera fehaciente el vínculo existente entre el acceso al agua y las condiciones de pobreza de la población.

Los resultados de las encuestas realizadas en la Comunidad El Cacao a los 24 jefes de familia que hacen uso de la fuente de agua en estudio entre finales del 2015 y mediados 2016, reportaron que existían un total de 123 personas que habitan en el lugar, de estas 66 personas adultas equivalen al 54\%; 53 son niños menores de edad y equivalen al $43 \%$ y solo se reportan 4 ancianos para un 3\% de adultos mayores (Figura 6). Basado en estos resultados se puede asumir que la esperanza de vida de las personas mayores en esta comunidad es baja. Según indicadores el promedio de esperanza de vida en Nicaragua está por los 75.10 años de vida para ambos sexos (Datosmacro, 2015). Lo cual no se cumple para el promedio de la población en estudio.

Con respecto a quienes están al frente de las familias, el estudio arrojó que los jefes de familia están compuestos mayoritariamente por mujeres con un 58\% (14 mujeres) y un 42\% (10 varones). La mayor concentración de las edades se da entre los rangos de 36 a 45 años con un 41,6\% (6 mujeres y 4 varones), 
lo que significa una composición de personas relativamente jóvenes, coincidiendo dicha información con la ya expresado anteriormente sobre la poca existencia de personas de la tercera edad.

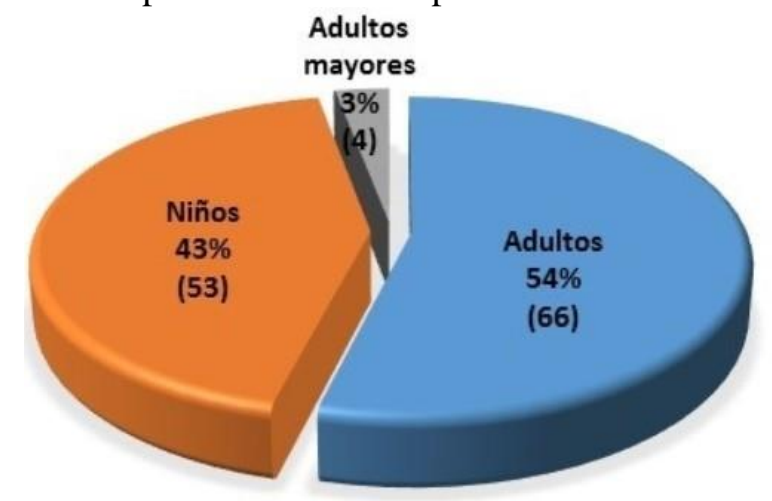

Figura 6. Representación demográfica de la cantidad de personas que habitan en la comunidad El Cacao y que son usuarios actuales de la fuente de agua en estudio.

Resultados referidos a ocupación, estado civil y nivel educativo de la población: La historia del mundo está llena de tensiones provocadas por la escasez de recursos naturales: oro, diamantes y petróleo. Sin embargo, el agua, que hasta ahora no había sido considerada un factor de conflicto, con el cambio climático se convierte en "el oro azul" de este siglo. El aumento de la población, inadecuadas políticas de gestión, falta de tecnología de punta y prácticas de corrupción agravan la situación, poniendo en peligro el desarrollo de la región latinoamericana (Nieto, 2011). Por otra parte, el analfabeto tiene mayores dificultades de inserción social no sólo a nivel personal (problemas de inclusión social, trabajo precario, altas morbilidades, etc.), sino también a nivel de su grupo familiar (nutrición, higiene, salud y escolaridad de los hijos, entre otros), como al resto de la sociedad (perdidas de productividad, altos costos para el sistema de salud) (Martínez, et al., 2014).

Según los resultados mostrado por la Tabla 2, las actividades que realizan los jefes de familia de la comunidad El Cacao (10 varones) se concentran mayormente en las labores agrícolas para su sobrevivencia. En su condición de campesinos se ocupan de la cría de animales, la transformación y comercialización de alimentos y otros productos y servicios derivados del agro. En el caso de las mujeres (14 mujeres) en su mayoría son amas de casa y sobre ellas recae la tarea de acarrear el agua desde la fuente hasta los hogares. Con respecto al estado civil 21 jefes de familia están casados, existen dos solteros y un viudo. Según los datos reportados también en la Tabla 2 el nivel educativo en esta comunidad es bajo, ya que son 7 los jefes de familia que no tienen nada de instrucción (debe entenderse que no saben leer y escribir), hay 9 alfabetizados, es decir que pocas personas saben leer y escribir y solo 8 personas han cursado máximo hasta el segundo grado de escolaridad.

Tabla 2. Ocupación, estado civil y nivel educativo de los jefes de familia de la comunidad El Cacao.

\begin{tabular}{lclcll}
\hline \multicolumn{1}{c}{ Ocupación } & \multicolumn{2}{c}{ Estado civil } & \multicolumn{2}{c}{ Nivel educativo } \\
\hline Agricultor & 10 & Casado & 21 & Ninguno & 7 \\
Doméstica & 1 & Soltero & 2 & Alfabetizado & 9 \\
Ama de casa & 13 & Viudo & 1 & $2^{\circ}$ grado de primaria & 8 \\
\hline
\end{tabular}

Según el informe Nicaragua en cifras que publicó el Banco Central de Nicaragua, BCN (2017), revela que de 3895447 personas que conforman la población mayor de diez años, 784174 equivalentes al 20,1\% no tiene ningún grado de instrucción académica. La estadística específica que de estos 784174 iletrados absolutos, 397501 son mujeres y 386673 son hombres; sin embargo, la cantidad de analfabetos podría ser mayor ya que 644440 personas sólo cursaron entre el primero y tercer grado de primaria, por lo que se les 


\section{L.P. Páramo-Aguilera y P. Rivas-Pérez}

considera analfabetos funcionales ya que apenas saben leer y escribir. El analfabetismo en la comunidad El Cacao hace que las condiciones de vida de las personas sean aún más precarias, no solo porque no pueden optar a trabajos mejor remunerados, sino por la poca información que pueden tener para mejorar sus sistemas de salubridad. Según Martínez y Fernández, 2010, el analfabetismo en la adultez no solo afecta en los conocimientos de sus derechos, sino que también será efecto de menores conocimientos de salud e higiene para el cuidado personal y el de la familia, los cuales se traducirán en una nutrición y salud deficientes.

Las personas que se encuentran en situación de analfabetismo o con conocimientos muy básicos en lectura y escritura enfrentan grandes dificultades para comprender y, consecuentemente, para poner en práctica, mensajes destinados a fomentar conductas saludables y de prevención de riesgos en diversos ámbitos de la vida cotidiana (Dexter, LeVine y Velasco, 1998). Investigaciones han sostenido, además, que se provocan limitaciones en los conocimientos y prácticas en torno al autocuidado, especialmente el de las mujeres, desencadenando problemas en la salud, higiene y nutrición de su hogar (UNESCO, 2006). Los trabajos presentados por Martinez y Fernández, 2010, UNESCO (2006) y considerando las cifras dadas por el Banco Central de Nicaragua, (BCN, 2017); nos muestran que la precaria condición de vida que se puede observar en la población de la comunidad El Cacao, está estrechamente relacionada con su bajo nivel educativo. De ahí que les sea sumamente difícil asimilar la importancia que tiene para la salud de los mismos, las diferentes medidas higiénicas que se requieren en el hogar y que a su vez se reflejan en el uso y manejo que hacen del escaso recurso agua con el que cuentan (desde el punto de recolección hasta el manejo en el hogar). Finalmente, las ocupaciones que realizan están directamente relacionadas con el bajo nivel de escolaridad, dedicándose la mayoría de ellos a las labores agrícolas y domésticas, razón por la cual sus ingresos son sumamente bajos y su condición de pobreza extrema se pone de manifiesto.

Ingreso mensual por jefe de familia y número de personas que habitan por vivienda: En las familias de escasos recursos, es frecuente que se privilegie el trabajo antes que la educación, debido al costo de oportunidad. La necesidad imperiosa de salir a trabajar para aportar a la subsistencia del hogar es la principal causa de deserción escolar en los jóvenes. A ello se suma la tentación de dejar el sistema escolar pues se percibe que no recompensa de manera proporcional la inversión en la culminación de sus estudios con sueldos y perspectivas laborales. Esto hace que los jóvenes de escasos recursos sean los más propensos a dejar la escuela. Los jóvenes que no alcanzan el certificado de educación primaria tienen menor probabilidad de tener empleos de calidad suficiente para mantenerse fuera de la situación de la pobreza (Marinho, 2007).

Con respecto al ingreso mensual expresado en la Figura 7, el más alto salario reportado se encuentra en el rango de 3001 (104.86 dólares) a más córdobas mensuales, aunque solo un 4,2\% lo reporta (Un caso). La menor cantidad está en el rango de 500 (17.48 dólares) a menos córdobas mensuales y lo reportan un 8,3\% (Dos casos). La mayor cantidad de jefes de familia percibe un ingreso que está entre los 1001 (34.98 dólares) a 1500 (52.41 dólares) córdobas con un 37,5\% equivalente a nueve casos, seguido por el rango de 2501 (87.39 dólares) a 3000 (104.83 dólares) córdobas con un porcentaje de 20,8\% (Cinco casos). La situación antes descrita convierte a esta comunidad en familias en extrema pobreza y alto riesgo alimentario, considerando el costo de vida según el valor de la canasta básica que a la fecha supera los 13000.00 (trece mil córdobas a junio del 2016) (454.23 dólares) para una familia promedio de 4 personas. La presencia de enfermedades relacionadas con la mala calidad del agua por la presencia de hongos y bacterias (PáramoAguilera et al., 2018), en conjunto con deficiencias alimentarias y la falta de acceso a sistemas de salud, implican que los pobladores de esta comunidad vean limitadas sus posibilidades de llevar a cabo actividades generadoras de ingresos que les permita sustentar sus precarios niveles de vida. El no poder asistir a un trabajo o desarrollar alguna actividad económica por causa de enfermedades, o la menor productividad que implica el trabajar en condiciones de salud precaria, conforma el llamado círculo vicioso entre agua y pobreza (OMS, 2016).

Nexo Revista Científica / Vol. 32, No. 01, pp. 27-40/Junio 2019 


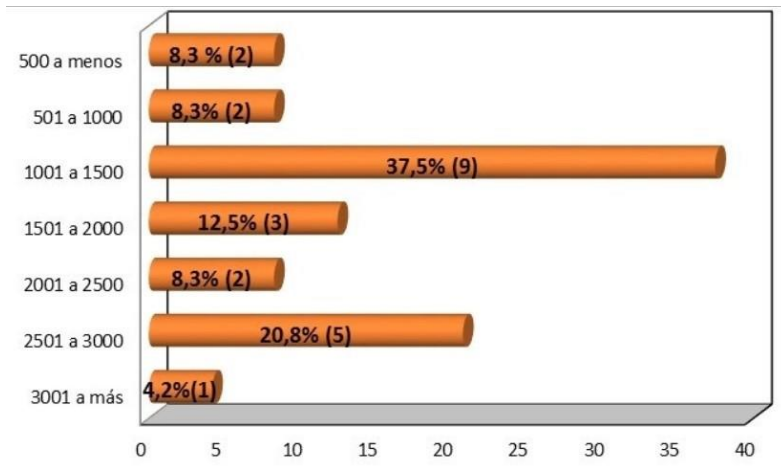

Figura 7. Ingreso económico mensual que tienen los jefes de familia de la comunidad El Cacao.

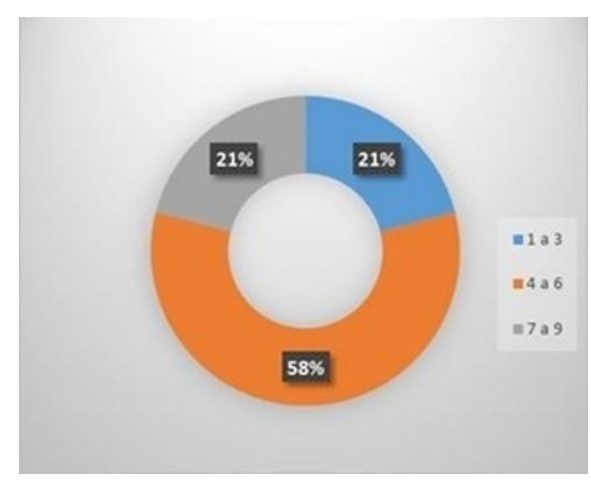

Figura 8. Cantidad de personas que habitan por casa en la comunidad El Cacao

Con respecto al número de personas que habitan por vivienda, en la Figura 8 se muestra la cantidad de personas que habitan en una casa, que está mayormente concentrado de 4 a 6 personas con un porcentaje de $58 \%$ (14 viviendas), además existe un promedio de 7 a 9 personas por familia correspondiente al $21 \%$ (5 viviendas) y un promedio de 1 a 3 personas con un porcentaje de $21 \%$ equivalente a 5 viviendas respectivamente. Los resultados descritos anteriormente indican que los pobladores de esta comunidad viven en hacinamiento debido a que la densidad poblacional por casa es alta. Durante la realización de las encuestas se pudo observar que las viviendas son pequeñas y viven en condiciones de vida precarias. Según Lentini y Palero (2009), el hacinamiento provoca que las personas se vean afectadas no únicamente por la incomodidad de tener que compartir un espacio mínimo, sino que también porque es prácticamente imposible mantener los hogares higiénicos afectando claramente la salud de las personas. Es por eso que los pobladores afirman el padecimiento de enfermedades relacionadas tanto a la mala calidad del agua y posiblemente al hacinamiento.

Resultados con respecto al número total de personas que trabajan por vivienda y características de esas viviendas: Según los resultados mostrados en la Tabla 3, se aprecia que en la comunidad El Cacao trabajan un $36,5 \%$ equivalente a 45 personas, es decir que, del total de 66 adultos, solamente 45 personas son el sostén de sus hogares. En 10 familias solamente trabaja una persona por familia, en 7 familias trabajan dos personas en cada una de ellas y en las otras 7 familias restantes trabajan hasta tres personas por núcleo familiar. Esto indica la relación existente entre los bajos ingresos económicos (Figura 7) y el analfabetismo en los pobladores de esta comunidad (Tabla 2), ya que no pueden optar a trabajos con mayores ingresos por lo que se dedican mayormente a las labores agrícolas para poder sobrevivir. Los adultos analfabetos, ya sea porque no tuvieron acceso a la educación formal, porque la abandonaron tempranamente para incorporarse al mercado de trabajo o que por desuso perdieron la capacidad de leer y escribir, poseen grandes limitaciones de empleabilidad debido a un nivel bajo de conocimientos y especialización. A ello se suma, que el individuo analfabeto tiene mayores limitaciones para conocer sus derechos y deberes, situación que puede derivar en la aceptación de contratos precarios y de baja calidad. Lo anterior aumenta la probabilidad de que dichas personas se mantengan en la pobreza (Martínez y Fernández, 2010).

Tabla 3. Cantidad de personas que trabajan por casa, según el total de familias encuestadas y total de personas que tienen un empleo remunerado.

\begin{tabular}{lcc}
\hline Por cada casa & $\mathbf{N}^{\mathbf{0}}$ de familias & Personas en total \\
\hline Una & 10 & 10 \\
Dos & 7 & 14 \\
Tres & 7 & 21 \\
Gran total & 24 familias & 45 personas \\
\hline
\end{tabular}


En la Tabla 4 se muestran los resultados de la caracterización física de las viviendas, donde un 91,7\% (22 casas) presentan las condiciones mínimas necesarias para ser llamadas casas, el 8,3\% (2 casas) son improvisadas. La Declaración Universal de Derechos Humanos (Arto. 25) reconoce el derecho de toda persona a un nivel de vida adecuado, incluyendo una vivienda digna y segura (ONU, 1948). Según un artículo publicado en La Prensa en julio del 2016 se dice: para que una vivienda sea considerada saludable debe estar construida con buenos materiales y no con desechos reciclables, y al menos deben de tener piso, paredes de concreto, techo y principalmente el acceso a servicios básicos como agua potable, energía y servicios higiénicos (letrinas) (Álvarez, 2016). La encuesta reflejó que en la comunidad El Caco, con respecto al tipo de techo hay un porcentaje de $87,5 \%$ ( 21 casas) que tienen techo de zinc, un 8,3\% (2 casas) tienen techo de tejas y solamente un 4,2\% equivalente a una casa tiene techo de paja. Aunque el $87,5 \%$ (21 casas) tienen techo de zinc, el 87,5\% (casas) presentan paredes de taquezal (sistema de construcción fundamentalmente con madera o palos de cañas entretejidos y barro, rellenos de tierra), un 8,3\% (2 casas) las paredes son de madera y solamente un $4,2 \%$ equivalente a una casa (Funciona como centro de Salud) las paredes son repelladas, lo que hace que sean casas muy inseguras y vulnerables ante los embates de la naturaleza, incluyendo también que el 91,7\% (22 casas) el tipo de piso es de tierra, solamente el 8,3\% equivalente a dos casas son embaldosadas. En la Figura 9 se puede apreciar las características físicas de las viviendas observadas durante las encuestas realizadas en la comunidad El Cacao.

Tabla 1. Resumen de la caracterización física de las viviendas de la comunidad El Cacao (tipo de vivienda, techo, paredes y piso).

\begin{tabular}{|c|c|c|c|c|c|}
\hline \multicolumn{2}{|c|}{ Tipo de vivienda } & \multicolumn{2}{|c|}{ Tipo de techo } & Tipo de paredes & Tipo de piso \\
\hline Casa & $91,7(22)$ & Zinc & $87,5(21)$ & Repellada $4,2(1)$ & $\begin{array}{ll}\text { Tierra } & 91,7(22)\end{array}$ \\
\hline Improvisada & $8,3(2)$ & $\begin{array}{l}\text { Tejas } \\
\text { Paja }\end{array}$ & $\begin{array}{l}8,3(2) \\
4,2(1)\end{array}$ & $\begin{array}{lc}\text { Madera } & 8,3(2) \\
\text { Taquezal } & 87,5(21)\end{array}$ & Embaldosada 8,3 (2) \\
\hline
\end{tabular}
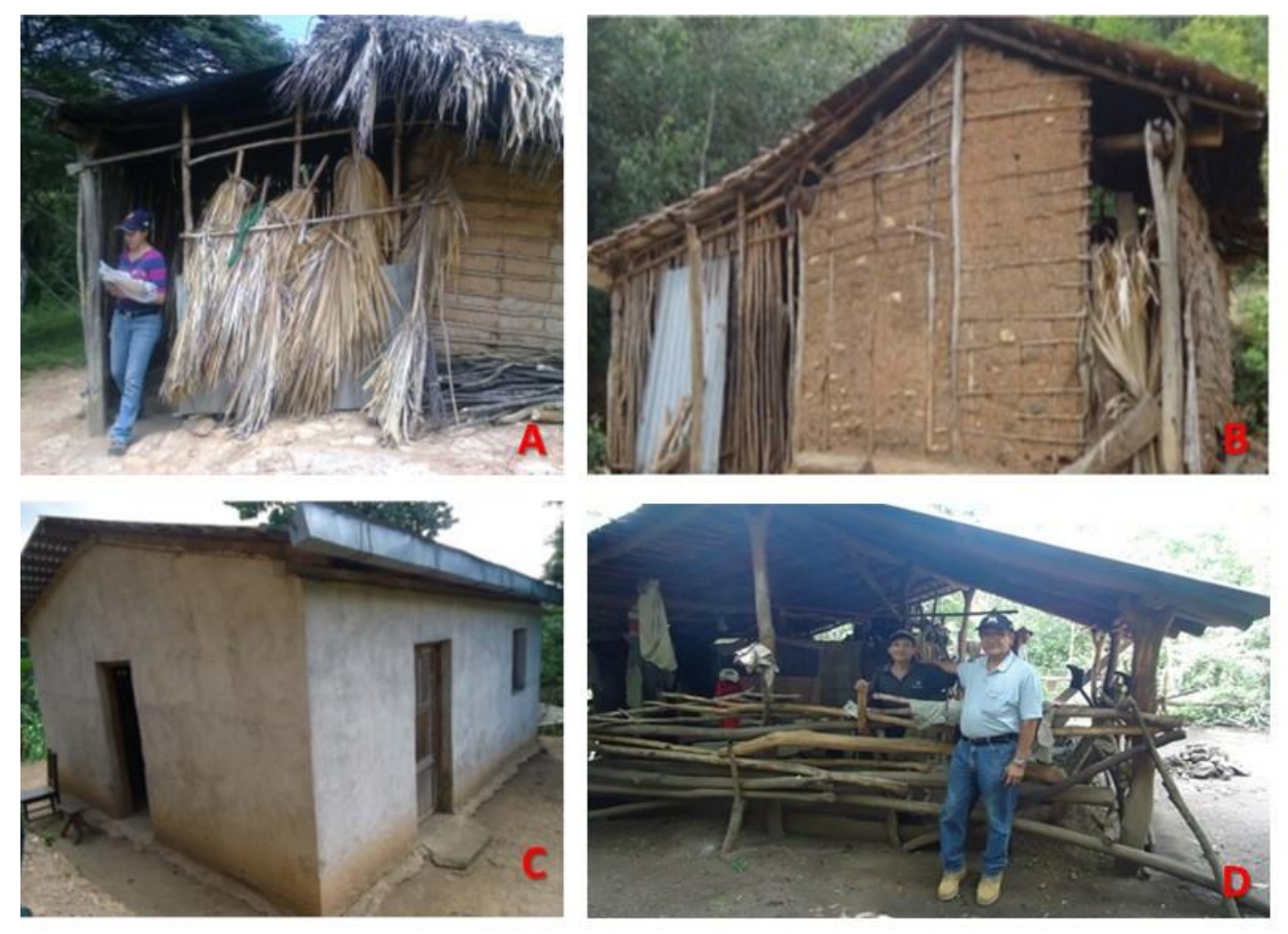

Figura 9. Fotografías de las viviendas de la Comunidad El Cacao. (A-B) Casas con paredes de taquezal, una con techo de palma y otra con techo de teja. (C) La única casa con paredes repelladas y que a su vez funciona como centro de salud. (D) Casa improvisada en donde habita la persona que donó el actual acuífero del que hace uso la comunidad. 
Resultados con respecto al manejo y consumo de agua de los habitantes de la comunidad El Cacao: El agua es esencial para la vida y todas las personas deben disponer de un abastecimiento satisfactorio (suficiente, seguro y accesible). La mejora del acceso al agua de consumo humano puede proporcionar beneficios tangibles para la salud. Se debe hacer el máximo esfuerzo para lograr que el agua de consumo humano sea tan segura como sea posible. El acceso al agua potable es fundamental para la salud, uno de los derechos humanos básicos y un componente de las políticas eficaces de protección de la salud (OMS, 2018).

La Figura 10 muestra el tipo de recipientes (Pichingas y bidones) utilizados por los pobladores de la comunidad El Cacao para trasladar el agua desde la fuente a sus casas de habitaciones. Un $67 \%$ equivalente a 16 familias hacen uso de las pichingas, mientras que el 33\% equivalente a 8 familias utilizan bidones. Con respecto al almacenamiento del agua, los pobladores emplean: barriles plásticos de 52 galones, pichingas y bidones, cabe destacar que solamente un porcentaje de $4 \%$ equivalente a una familia hace uso de los barriles plásticos, un porcentaje de $40 \%$ equivalente a 10 familias utilizan pichingas, el 56\% restante (13 familias) almacenan el agua en bidones. Esto nos da una idea del poder adquisitivo de la población.
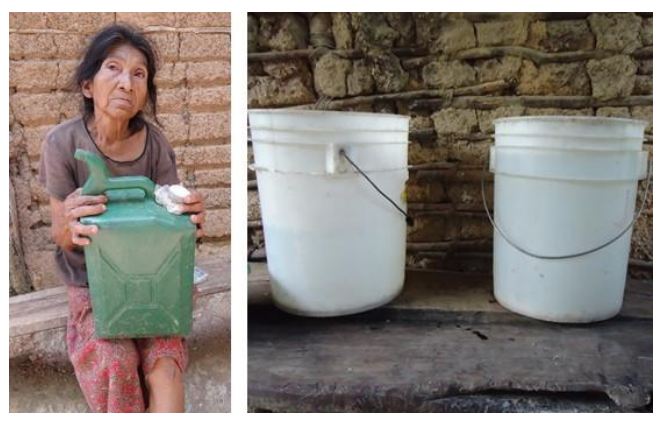

Figura 10. Fotografías de los recipientes donde trasladan el agua desde la fuente a los hogares. (A) Pichingas utilizadas comúnmente por ser más fáciles de llevar. (B) Bidones usados por los pobladores para llevar un mayor volumen de agua a sus hogares y que a su vez sirve para almacenarla en casa.

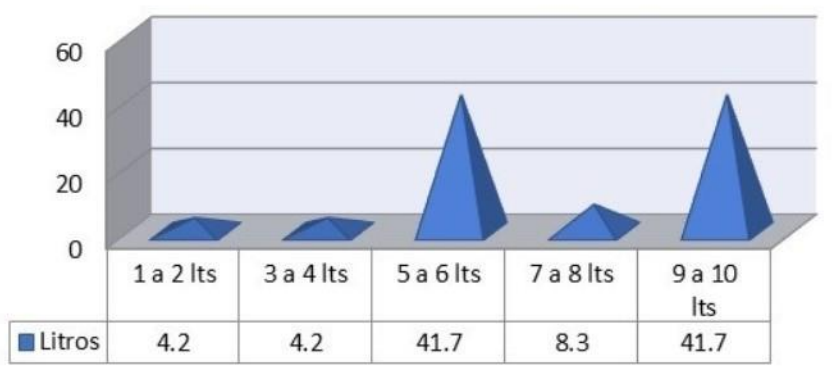

Figura 11. Representación gráfica de la cantidad promedio de agua diaria consumida por familia en la comunidad El Cacao

Una vez que los pobladores de la comunidad El Cacao trasladan el agua de la fuente hasta sus hogares, 21 familias preservan el agua utilizando las mismas tapas de los recipientes, una familia reporta que no tapa el agua, mientras que dos familias refieren que utilizan otros medios para tapar el agua (Plástico y tela) y así evitar que esta se contamine. Se pudo establecer existe un 87,5\% (21 familias) que separan el agua de tomar de la que usan para las labores domésticas, el 12,5\% (3 familias) no separan el agua, también se pudo observar que solamente el 70,8\% equivalente a 17 familias de los que separan el agua, le dan algún tratamiento de purificación (Filtración, hervido o cloración fundamentalmente). Lo anterior muestra que un 29,2\% (7 familias) consumen el agua sin ningún tipo de tratamiento y están en riesgo directo de adquirir algún tipo de infección provocada por la presencia de hongos y bacterias en el agua (Páramo-Aguilera et al., 2018). Es aún más preocupante el hecho que de las 17 familias que hacen uso de algún proceso de purificación, 11 de ellas utilizan filtro artesanal (donados en algún momento por organismos cooperantes, pero que ya están obsoletos). Dos familias reportan el uso de cloro para purificar el agua (Sin tener claridad de la dosis a utilizar por litro de agua), las cuatro familias restantes afirman que hierven el agua antes de ingerirla.

Según la OMS (2007), la desinfección del agua en los hogares puede ser una opción de bajo costo. La desinfección solar es gratuita, a condición de disponer de botellas de plástico. Cuesta muy poco preparar una disolución de cloro y se pueden utilizar filtros de cerámica sencillos, moldeados por artesanos locales. El hervor es, con mucho, el método más utilizado para desinfectar el agua en el hogar. La implementación 


\section{L.P. Páramo-Aguilera y P. Rivas-Pérez}

de algunas de estas técnicas en los hogares de la comunidad El Cacao ayudaría a mantener la salubridad del agua.

Según La figura 11 la mayor concentración de ingesta de agua por familia en la comunidad El Cacao se concentra entre 9 a 10 litros al día con un 41,7\% equivalente a 10 familias, seguido de una ingesta de 5 a 6 litros en la misma proporción (41,7\%) correspondiente a 10 familias. Un 8,3\% (2 familias) consumen de 7 a 8 litros de agua diariamente y un porcentaje del 8,3\% consumen entre 1 a 4 litros de agua al día (2 familias). La OMS (2016), señala que la ingesta de agua por persona está comprendida entre 2 a 2,5 litros de agua. Según la figura antes citada, las 24 familias que hacen uso del acuífero en estudio de la comunidad El Cacao presentan un consumo promedio de agua para tomar de 182 litros en total por día. Por tanto, considerando que son 123 personas en la comunidad, se estima que tienen una ingesta de 1,47 L/hab/día, es decir que los habitantes de la comunidad El Cacao tienen una ingesta de agua menor que la señalada por la OMS.

Finalmente se pudo establecer que, con respecto al consumo de agua para las labores domésticas, es de 770 litros por día para toda la comunidad, es decir un promedio de 6,26 L/hab/día. Según el órgano rector del consumo de agua (ENACAL), las dotaciones de consumo de agua de uso doméstico para zonas rurales con un rango de población de 0 a 5000 habitantes deber ser de $75 \mathrm{~L} /$ hab/día, es decir que los pobladores de esta comunidad utilizan una cantidad de agua que es unas doce veces inferior a lo establecido en el país, razón que deberá ser de gran preocupación para las autoridades competentes y así llevar agua a esta comunidad en cantidad y calidad suficiente que les permita mejorar sus condiciones de vida.

\section{CONCLUSIONES}

Los resultados de las encuestas aplicadas a 24 jefes de familia de la comunidad El Cacao, indicaron la relación que existe entre la educación sanitaria y la calidad del agua, ya que los pobladores carecen del conocimiento de normas higiénico sanitarias orientadas al mejoramiento de las condiciones de vida y que están estrechamente relacionadas con el manejo y cuido del agua de consumo. También se evidenció la relación existente entre los bajos niveles educativos encontrados en la zona y los bajos niveles de ingreso por familia que fundamentalmente se dedican a labores agrícolas y domesticas; entre el número de miembros de la familia y condiciones de vida observadas en el hogar; entre el nivel de ingreso por familia, las condiciones físicas de la vivienda y las condiciones de salud observadas con el manejo del agua desde el acuífero hasta el hogar y dentro del mismo. Todo ello corroboró la estrecha relación que existe entre nivel de vida en la zona y los niveles educacionales con relación al manejo y acceso al agua de consumo en cantidades y calidades suficientes.

\section{REFERENCIAS}

Álvarez, W. (09 de julio del 2016). Tres de cada 10 nicas con casas inadecuadas. La Prensa. Recuperado de: $\quad$ http://www.laprensa.com.ni/2016/07/09/economia/2065378-tres-10-nicas-casas-inadecuadas. Consultado en mayo de 2017.

Banco Central de Nicaragua (BCN). (2017). Nicaragua en cifras 2017. ISSN 2410-8405 (edición electrónica).

Recuperado de: https://www.bcn.gob.ni/publicaciones/periodicidad/anual/nicaragua cifras/nicaragua cifras.pdf. Consultado el 20 de septiembre del 2018.

Datosmacro. (2015). Esperanza de vida en Nicaragua. Recuperado de:http://www.datosmacro.com/demografia/esperanzavida/nicaragua. Consultado en mayo de 2017.

Dexter, E., LeVine, S \& Velasco, P. (1998), Maternal schooling and health-related language and literacy skills in rural Mexico. Comparative education review, 42 (2), 139-162.

Nexo Revista Científica / Vol. 32, No. 01, pp. 27-40/Junio 2019 
Garmendia-Almendarez, T.S y Villalta-Domínguez, J.L. (2017). Evaluación y propuesta de mejora del sistema de captación y conducción del agua de consumo, comunidad El Cacao. Mozonte, Nueva Segovia. Trabajo de diploma para optar al título de Ingeniero Químico, presentado a la Facultad de Ingeniería Química, de la Universidad Nacional de Ingeniería (UNI). Managua, Nicaragua, en Julio del 2017.

Instituto Nacional de Información de Desarrollo [INIDE]. (2010). Departamento de Nueva Segovia y sus municipios. Uso de la tierra y el agua en el sector agropecuario. Recuperado de: http://www.magfor.gob.ni. Consultado el 20 de Octubre del 2018.

Lentini, M y Palero, D. (2009). El hacinamiento: la dimensión no visible del déficit habitacional. Revista INVI, 12(31).

Martínez, R., Trucco, D y Palma, D. (2014). El analfabetismo funcional en América Latina y el Caribe. Panorama y principales desafíos de política. Serie Políticas Sociales. Naciones Unidas, CEPAL (Comisión Económica para América Latina y el Caribe). ISSN 1564-4162. LC/L.3841. Santiago de Chile.

Marinho, M.M.L. (2007), El eslabón perdido entre educación y empleo. Análisis sobre las percepciones de los jóvenes urbanos de escasos recursos en Chile, Políticas Sociales, CEPAL, Santiago de Chile. ISSN versión impresa 1564-4162 ISSN LC/L.2783-P.

Martínez, R y Fernández, A. (2010). Impacto social y económico del analfabetismo: Modelo de análisis y Estudio Piloto. Documento de Proyecto. Naciones Unidas, CEPAL (Comisión Económica para América Latina y el Caribe). LC/W.299. Oficina Regional de Educación para América Latina y el Caribe. UNESCO. Santiago. UNE/08/001. Santiago de Chile.

McJunkin, E. (1986). Agua y Salud Humana. México. Primera edición Organización Panamericana de la Salud (OPS). Editorial Limusa, S.A. pag.22. Sección 1.

Nieto, N. (2011). La gestión del agua: Tensiones globales y latinoamericanas. Política y cultura. 36, pp.157.176.

Organization for economic co-operation and development [OECD]. (2003). Social issues in the provision and pricing of eater services. Recuperado de: https://www.oecd.org/env/resources/15425332.pdf. Consultado el 20 de octubre del 2018.

Organización Mundial de la Salud [OMS]. (2006). Guías para la calidad del agua potable. Primer Apéndice a la tercera edición. Recomendaciones. Volumen 1. ISBN 924154696 4. Clasificación de la NLM: WA 675.

Organización Mundial de la Salud [OMS]. (2007). Lucha contra las enfermedades transmitidas por el agua en los hogares. Red internacional para la promoción del tratamiento y el almacenamiento seguro del agua doméstica. ISBN 978924359522 1. Clasificación NLM: WA 675.

Organización Mundial de la Salud [OMS]. (2016). Agua, saneamiento e higiene para acelerar y sostener el progreso respecto de las enfermedades tropicales desatendidas. Una estrategia mundial 2015-2020.

Organización Mundial de la Salud [OMS]. (2018). Guías para la calidad del agua de consumo humano. Cuarta edición que incorpora la primera adenda. World Health Organization. Ginebra, 2011. 
Organización de las Naciones Unidas [ONU]. (1948). Declaración Universal de los Derechos Humanos. Recuperado en: http://www.ohchr.org/EN/UDHR/Documents/UDHR Translations/spn.pdf. Consultado en mayo de 2017.

Páramo-Aguilera, L.A., Garmendia, T.S y Villalta-Dominguez, J. (2018). Herramientas moleculares para estudiar las aguas de consumo humano del Cacao, Mozonte, Nueva Segovia, Nicaragua. Revista científica NEXO, 31(01), pp. 01-15. ISSN-E 1995-9516. Universidad Nacional de Ingeniería http://revistas.uni.edu.ni/index.php/Nexo, http://dx.doi.org/10.5377/nexo.v31i01.6450 .

Pérez, L. (2007). Los derechos de sustentabilidad: Desarrollo, consumo y ambiente. Argentina, Primera Edición. Editorial Colihue, pp.1-287.

UNESCO. (2006), Why literacy matters. In UNESCO (Ed.), Education for all. Literacy for life , pp. 135145). Paris: Unesco Publishing.

Valiente, C y Mora, D. (2002). El papel del agua para consumo humano en los brotes de diarrea reportados en el período 1999 - 2001 en Costa Rica. Rev. costarric. salud pública, 11 (20). pp.1-13. ISSN 1409-1429.

\section{SEMBLANZA DE LOS AUTORES}

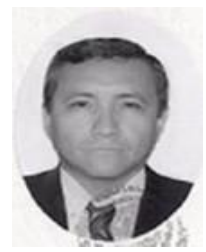

Leandro Alberto Páramo Aguilera, Graduado como Ingeniero Químico con maestría en Ingeniería Química y énfasis en procesos biotecnológicos, en el Instituto Superior Politécnico "José Antonio Echeverría", ISPJAE, de la Ciudad de la Habana, Cuba, en el año 1990. En 1997 se gradúa como Master en Microbiología y énfasis en bacteriología en la Universidad de Costa Rica, UCR. En junio del 2012, se gradúa como Doctor en Ciencias en el área de Biotecnología en el Centro de Biotecnología Genómica (CBG) del Instituto Politécnico Nacional (IPN) de México. Amplia experiencia en el desarrollo de procesos biotecnológicos (biofertilizantes, bebidas alcohólicas, fermentados lácteos, bioprospección, compostaje, etc).

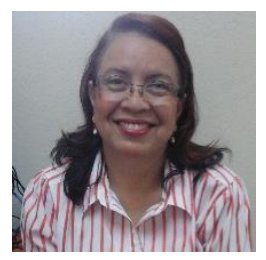

Patricia Rivas Pérez, Graduada como Licenciada en Biología en la Universidad Nacional Autónoma de Nicaragua en León (UNAN-León) en el año 1990, con maestría en Administración y Dirección de Empresas con énfasis en Mercadeo, de la Universidad Centroamericana (UCA) Managua, en el año 2000. Docente horario de la Universidad Centroamericana. Amplia experiencia en Investigación de Mercados, procesamiento estadístico de información trabajando con software computacionales, etc. 